\title{
Development of an optimized conditionally replicative adenoviral agent for ovarian cancer
}

\author{
ZENG B. ZHU ${ }^{1}$, BAOGEN LU ${ }^{1}$, MIEY PARK ${ }^{1}$, SHARMILA K. MAKHIJA ${ }^{2}$, THOMAS M. NUMNUM ${ }^{2}$, \\ JAMES E. KENDRICK ${ }^{2}$, MINGHUI WANG ${ }^{1}$, YUKO TSURUTA ${ }^{1}$, PAUL FISHER ${ }^{5}$, RONALD D. ALVAREZ ${ }^{2}$, \\ FEN ZHOU ${ }^{3}$, GENE P. SIEGAL ${ }^{4}$, HONGJU WU $^{1}$ and DAVID T. CURIEL ${ }^{1}$ \\ ${ }^{1}$ Division of Human Gene Therapy, Departments of Medicine, Pathology, Surgery, Ob/Gyn and the Gene Therapy Center, \\ ${ }^{2}$ Division of Gynecologic Oncology, ${ }^{3}$ Departments of Anesthesiology, ${ }^{4}$ Pathology, Cell Biology, and Surgery and the Gene \\ Therapy Center, University of Alabama at Birmingham, AL 35291; ${ }^{5}$ Department of Pathology, \\ Neurosurgery, and Urology, College of Physicians and Surgeous, Columbia University, NY, USA
}

Received November 7, 2007; Accepted January 24, 2008

\begin{abstract}
Human ovarian cancer is a highly lethal malignant neoplasm in woman with no effective treatment if conventional chemotherapy fails. In this regard, conditionally replicative adenoviruses (CRAds) represent a promising new modality for the treatment of cancer. A key contribution to the development of CRAds was the introduction of tumor-selective viral replication to restrict amplification to the neoplastic cell population. Under ideal conditions following cellular infection, the viruses replicate selectively in the infected tumor cells, killing the cells by cytolysis, leaving normal cells unaffected. However, to date, there have been limitations to the clinical application of these CRAd agents i.e. poor viral infectivity, poor tumor specificity and high toxicity. Here, we report the in vitro and in vivo comparison of four CRAd agents developed for ovarian cancer application, specifically, Ad- $\Delta 24 . F 5 / 3$, CRAd-C.F5/3, CRAd-M.F5/3 and CRAd-S.F5/3. All CRAd agents contained fiber knob chimeras of adenovirus serotype 3, which enhanced the viral infectivity at the transductional level via a non-Coxsackie-Adenovirus Receptor alternative pathway. In addition, these CRAds embodied distinct mechanisms for the achievement of replication specificity. Tumor cell killing was assessed by using an oncolytic assay and a cell viability assay (MTS) in vitro, while tumor growth
\end{abstract}

Correspondence to: Dr David T. Curiel, Division of Human Gene Therapy, Gene Therapy Center, BMR II 508, 901 19th Street S., University of Alabama at Birmingham, Birmingham, AL 35291, USA

E-mail: curiel@uab.edu

Abbreviations: bp, base pairs; CMV, cytomegalovirus; CRAd, conditionally replicating adenovirus; MOI, multiplicity of infection; pfu, plaque-forming units; vp, viral particles, TSP, tumor-specific promoter

Key words: adenovirus, conditionally replicative adenovirus, fiber modification, tumor specific promoter was examined in a xenograft model in vivo by using a bioluminescent imaging assay. In addition, the replication rates of the CRAd agents were determined in human liver slices. Both the Ad- $\Delta 24 . F 5 / 3$ and CRAd-S.F5/3 were demonstrated to have higher tumor killing effects in tumor cells and a lower viral replication rate in human liver. These agents are thus excellent candidates for clinical trials of CRAd agents against human ovarian cancer.

\section{Introduction}

Ovarian carcinoma is the fourth leading cause of cancer death in American women and the most common gynecological malignancy. In 2006, there were 20,180 new cases and approximately 15,310 women succumbed to this disease in United States (1). Despite advances in surgical techniques and modern chemotherapy, the long-term survival rate is still low and the majority of patients ultimately relapse (2-4). In this regard more effective treatment strategies are needed for this disease.

Viral therapy (virotherapy or viral oncolytic therapy) represents a novel investigational approach to treatment of ovarian cancer. In this therapeutic strategy target tumor killing by the viral agent is achieved as a direct consequence of viral replication (5). The most studied construct is the one originally generated by Barker and Berk (dl1520) (6) and used initially by the McCormick group as a selective vector, named ONYX-015 (7). This viral vector originally was believed to replicate only in p53-defective cells (present in $\sim 50 \%$ of solid human tumors), however, this mechanism has subsequently been questioned (8). Based on the significant antitumor activity demonstrated using ONYX-015 both in vitro and in vivo, the preclinical potential of virotherapy has led to their rapid translation into human clinical trials, including those targeting recurrent head and neck (9), pancreatic (10), colorectal (11), ovarian (12) and hepatobiliary cancer (13). Another conditionally replicative adenovirus agent is the Ad5- $\Delta 24$, which contains a partial deletion in the CR2 domain of the pRb-binding protein E1A (d1919-943) which is complemented in pRb-deficient tumor 
cells (14). An integrin-directed infectivity-enhanced variant, CRAd- 124.RGD (15) has previously demonstrated effective killing of different cancer cells in vitro and in vivo (16-19). This agent is current being evaluated in a phase I clinical trail of ovarian cancer at UAB. An ideal virotherapy agent would possess 3 characteristics: i) high viral infectivity to tumor cells vs. non-tumor cells; ii) relative preference for replication in tumor vs. non-tumor cells; and iii) low replication rate in human liver tissue. However, both viral infectivity and specificity are poor in currently available conditionally replicative viral vectors.

The poor infectivity with current non-replicative and replicative type 5 Ad systems has been found to result from a relative paucity of the primary receptor for adenovirus type 5, the Coxsackie-Adenovirus Receptor (CAR), on the surfaces of tumor cells relative to their cell line counterparts $(20,21)$. On this basis, it has been proposed that gene delivery via 'CAR-independent' pathways may be required to circumvent this key aspect of tumor biology $(22,23)$. Many approaches have been described to enhance the viral infectivity by alternative vector tropism. Specifically, Dmitrev et al (24) reported that construction of modified adenoviral vectors containing the RGD peptide in the HI loop region, which targets the integrins $\alpha \mathrm{v} \beta 3$ and $\alpha \mathrm{v} \beta 5$ instead of CAR (25). Recently, other approaches have been reported which include targeting Ad to the serotype 3 receptor with a chimeric fiber protein $(26,27)$, and targeting Ad to a heparin sulfate-containing receptor via an $\mathrm{Ad}$ fiber incorporating polylysine (pK7) (28).

The improvement of tumor specificity with current replicative Ad systems has also recently been enhanced by using tumor specific promoters (TSPs) to drive adenoviral E1 expression, resulting in the viral replication being restricted in normal host cells, but not in tumor cells, thereby avoiding host toxicity by the CRAd agent. An ideal tumor specific promoter (TSP) for transcriptional targeting exhibits selective high activity in tumor cells (a 'tumor on'phenotype) and exhibits low activity in the endogenous sink, i.e. the liver (a 'liver off' phenotype). Many TSPs have been explored for specific cancers, such as prostate-specific antigen for prostate cancer, and $\alpha$-fetoprotein promoter for hepatocarcinoma $(29,30)$. However, there have been fewer reports of candidate TSPs for targeting ovarian cancer. In our laboratory, we have exploited several candidate ovarian cancer TSPs, including the Cox-2 (31), Midkine (Mk) (32), VEGF (33), SLPI (34), TERTS (35), CXCR4 (36) and survivin (37) promoters. Of note, many CRAd agents with these varying promoters have been screened in ovarian cancer patients tissues (38); albeit the transductional analysis and the toxicity analysis have not been previously fully examined or reported.

In our group, various candidate ovarian cancer CRAd agents with different TSPs and carrying different fiber modifications were generated, however, they have not yet been systematically tested in ovarian cancer cells in head-to-head manner. In this report, we have evaluated the potencies of a series of CRAd agents in ovarian cancer cell lines at the transductional and transcriptional levels and also examined human host cell toxicity both in vitro and in vivo. These data provide critical pre-clinical information and parameters necessary to ultimately conduce clinical trails.

\section{Materials and methods}

Cells and tissues. Human ovarian cancer cell lines, UCI101, SKOV3.ip1, HEY and OV4 were used in this study. SKOV3.ip1, HEY and OV4 were kind gifts from Dr Janet Price (The University of Texas M.D. Anderson Center, Houston, TX), Dr Judy Wolf (M.D. Anderson Center) and Dr Timothy J. Eberlein (Harvard Medical School, Boston, MA), respectively. The UCI101 cell line was purchased from ATCC. An imaging cell line, SKOV3Luc, was a kind gift from Dr Rober Negrin (Stanford Medical School, Stanford, CA) and 911 cells were obtained with gratitude from Dr Van Der Eb (Leiden University, The Netherlands). Nontransformed human skin fibroblasts were a kind gift from Dr Suresh Boppana (Childrens Hospital of Birmingham). Cells were maintained in Dulbecco's modified Eagle's medium. Each medium was also supplemented with $10 \%$ fetal calf serum, penicillin $(100 \mathrm{IU} / \mathrm{ml})$ and streptomycin $(100 \mu \mathrm{g} / \mathrm{ml})$. Cells were incubated at $37^{\circ} \mathrm{C}$ in a $5 \% \mathrm{CO}_{2}$ environment under humidified conditions.

Human liver samples were obtained from hepatectomy remnants not needed for diagnostic purposes, were collected by following IRB approval. To generate tissue slices, tissue was cut in consecutive $0.5-\mathrm{mm}$ thick slices using the Krumdieck tissue slicer (Alabama Research Development, Munford, AL). Sequential slices were then cultured in 24well plates in RPMI medium supplemented with $10 \%$ bovine fetal serum, $100 \mathrm{U} / \mathrm{ml}$ penicillin, $100 \mu \mathrm{g} / \mathrm{ml}$ streptomycin and $5 \mu \mathrm{g} / \mathrm{ml}$ insulin. Cultures were maintained at $37^{\circ} \mathrm{C}$ in a humidified atmosphere of $95 \%$ air and $5 \% \mathrm{CO}_{2}$. Three tissue slices were examined per group.

Recombinant adenoviruses. All recombinant adenoviruses including Ad5-CMV.Luc.F5/3, Ad5-CMV.Luc.RGD., Ad5CMV.Luc.pk7, Ad5-CMV.Luc.RGDpk7, and Adwt.F5/3, Ad- $\Delta 24 . F 5 / 3$ (deletion of nt $922-947$ in the E1 gene), CRAdM.F5/3 (M, the mesotheline promoter), CRAd-C.F5/3 (C, the CXCR4 promoter) and CRAd-S.F5/3 (S, the survivin promoter) were generated at UAB. The viruses were propagated in 911 cells, and purified by double $\mathrm{CsCl}$ density gradient centrifugation, followed by dialysis against phosphate-buffered saline (PBS) containing 10\% glycerol. The viruses were titrated by plaque assay in 293 cells, and vp number was determined spectrophotometrically based on absorbance at a wavelength of $260 \mathrm{~nm}$. The viruses were stored at $-80^{\circ} \mathrm{C}$ until use. The characteristics of all adenovirus vectors used in this study are shown in Table I.

Transductional evaluations in vitro. Transformed ovarian cells $\left(5 \times 10^{4}\right.$ cells/well), including UCI101, SKOV3.ip1, HEY and OV4, were plated on 24-well plates in $1 \mathrm{ml}$ of medium. The next day, cells were infected with recombinant Ads, Ad5.Luc, Ad5-CMV.Luc.F5/3, Ad5-CMV.Luc.RGD, Ad5CMV.Luc.pk7 or Ad5-CMV.Luc.RGDpk7, (Fig. 1) for transcriptional evaluation at $100 \mathrm{vp} / \mathrm{cell}$, for $2 \mathrm{~h}$, in $200 \mu \mathrm{l}$ of the medium containing $2 \%$ of FCS. Cells were then washed once with $1 \mathrm{ml}$ of PBS and $1 \mathrm{ml}$ of the medium containing $10 \%$ of FCS was added to each well. After 48-h incubation, cells were washed with PBS, luciferase activity was determined using the Reporter Lysis Buffer and the Luciferase 
Table I. The characteristics of adenoviral vectors used in this study.

\begin{tabular}{llllllc}
\hline Virus name & \multicolumn{1}{c}{ Promoter } & Reporter & E1 & E3 & Modification & Replication \\
\hline Ad5-CMV.Luc & CMV & Luciferase & No & No & No & No \\
Ad5-CMV.Luc.F5/3 & CMV & Luciferase & No & No & F5/3 & No \\
Ad5-CMV.Luc.RGD & CMV & Luciferase & No & No & RGD4C & No \\
Ad5-CMV.Luc.pk7 & CMV & Luciferase & No & No & pk7 & No \\
Ad5-CMV.Luc.RGDpk7 & CMV & Luciferase & No & No & RGDpk7 & No \\
Adwt.F5/3 & No & No & Yes & Yes & F5/3 & Yes \\
Ad- $\Delta 24 . F 5 / 3$ & No & No & L24 & Yes & F5/3 & Yes \\
CRAd-CXCR4.F5/3 & CXCR4 (C) & No & Yes & Yes & F5/3 & Yes \\
CRAd-Mesotheline.F5/3 & Mesotheline (M) & No & Yes & Yes & F5/3 & Yes \\
CRAd-Survivin.F5/3 & Survivin (S) & No & Yes & Yes & F5/3 & Yes \\
\hline
\end{tabular}

UC101

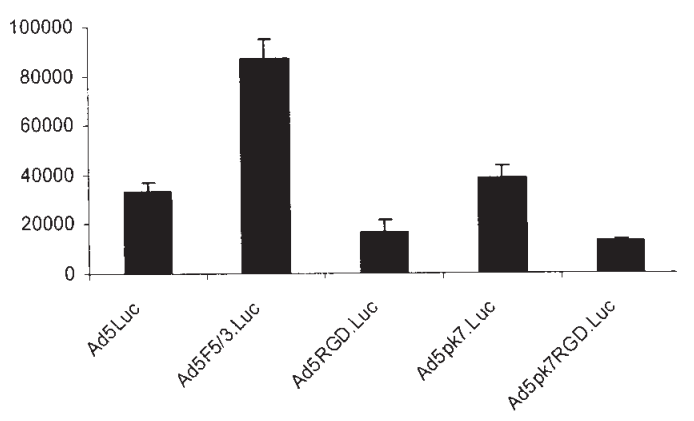

HEY

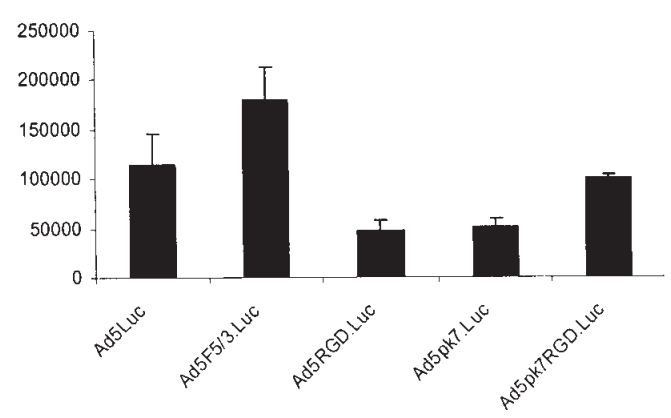

SKOV3ip1

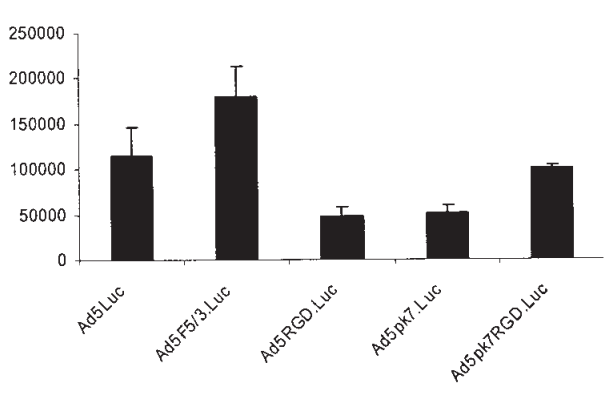

OV4

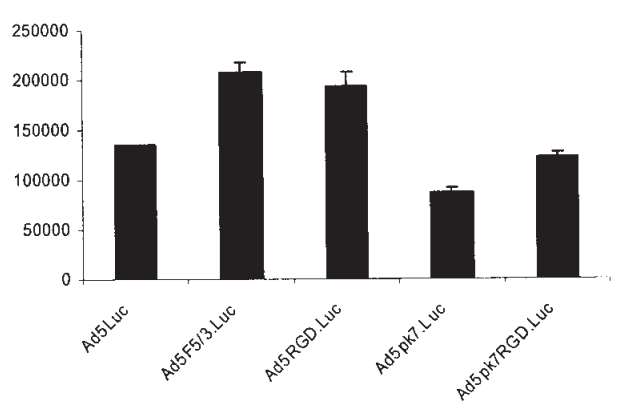

Figure 1. Comparison of tranductional activity in ovarian cancer cell lines with different capsid modified adenovirus vectors. Ovarian tumor cells $\left(5 \mathrm{x} 10^{4}\right)$ were plated on 24-well plates and infected at a MOI of 100 vp/cell of Ad5-CMV.Luc, Ad5.RGD.Luc, Ad5.pk7.Luc, Ad5.F5/3.Luc or Ad5.pk7.RGD.Luc, respectively. Luciferase activities were analyzed $48 \mathrm{~h}$ later. Results are shown as relative light units (RLU) of luciferase activity. The \% of luciferase activity $=($ RLU induced by TSP $) /($ RLU induced by the CMV promoter $) \times 100 \%$. The mean value \pm SE of triplicate samples is shown.

Assay System of Promega (Madison, WI) following the manufacturer's protocol. Experiments were performed in triplicate and luciferase activities were standardized to the relative light unit (RLU) values of the Ad5-CMV.Luc (the CMV promoter activity is set as $100 \%$ ). The transductional levels of the Ads in ovarian tumor cells were thus evaluated by expression activity of the luciferase reporter gene. Detected activities of the luciferase reporter gene were normalized by the expression activity of Ad5-CMV.Luc for the transductional level evaluations.

Cytocidal effects with staining assay. The in vitro cytocidal effect of the CRAd agents was analyzed by determining the viability of the test cells by crystal violet staining after infection. Briefly, 25,000 cells (UCI101, SKOV3.ip1, HEY and OV4) well were plated on 12 -well plates. Cells were infected at $100,10,1$, or $0 \mathrm{vp} /$ cell with Adwt.F5/3, Ad$\Delta 24 . F 5 / 3$, CRAd-M.F5/3, CRAd-C.F5/3 or CRAd-S.F5/3 in infection medium. Two hours later, the infection medium was replaced with the appropriate complete medium. After 10 days of cultivation, the cells were fixed with $10 \%$ buffered formalin for $10 \mathrm{~min}$ and stained with $1 \%$ crystal violet in $70 \%$ ethanol for $20 \mathrm{~min}$, followed by washing 3 times with tap water and air drying. Trypan blue exclusion experiments were also performed as described elsewhere (39). 
Determination of cell viability (MTS assay). A CellTiter 96 Aqueous One Solution Cell Proliferetion Assay was purchased from Promega (Madison, WI) and used according to the recommendations of the manufacturer. Briefly, $1 \times 10^{4}$ cells (UCI101, SKOV3ip1, HEY or OV4) well were plated on 96-well plates. Cells were infected at $0,1,10,100$ or 1,000 vp/cell with Adwt.F5/3, Ad- $\Delta 24 . F 5 / 3$, CRAd-M.F5/3, CRAd-C.F5/3 or CRAd-S.F5/3 in infection medium. Three hours later, the infection medium was replaced with the appropriate complete medium without phenol red. After a 4-day incubation, at $37^{\circ} \mathrm{C}$, with $5 \% \mathrm{CO}_{2}, 20 \mu \mathrm{l}$ of the assay reagent [3-(4,5-dimethylthiazol-2-yl)-5-(3-carboxy-methoxyphenyl)-2-(4-sulfophenyl)-2H-tetrazolium, inner salt] was added to each well that contained $100 \mu \mathrm{l}$ of medium. Cells were further cultured for $3 \mathrm{~h}$ and the resultant absorbance was record at $490 \mathrm{~nm}$ using a 96-well plate reader. Each experiment was repeated three times.

In vivo bioluminescent imaging. Thitry-five BALB/c nude mice were used in this study (5 mice per group). SKOV3Luc cells were cultured by conventional methodologies and cell viability was determined to be $>98 \%$ by using Trypan blue exclusion techniques. SKOV3Luc cells $\left(1 \times 10^{7}\right)$ were inoculated i.p. and tumor grew 5-day post injection. vp Ad vectors $\left(1 \times 10^{9}\right)$ including Adwt.F5/3, CRAd- 424.F5/3, CRAd-C.F5/3, CRAd-M.F5/3, CRAd-S.F5/3 and PBS control, in $200 \mu 1$ volume were injected i.p. The injection was performed once a week for 3 weeks. After inoculation and before treatment, bioluminescent imaging was determined as following: mice were placed in the imaging chamber and maintained with 2\% isoflurane (Minrane Inc., Bethlehem, PA) gas anesthesia at a flow rate of approximately $0.5-11 / \mathrm{min}$ per mouse (Highland Medical Equipment, Temecula, CA). Anesthetized mice were injected intraperitoneally with $150 \mathrm{mg} / \mathrm{kg}$ luciferin (Xenogen Corporation, Alameda, CA). After 10-20 min, mice were imaged using a charge-coupleddevice (CCD) camera completed to the Xenogene IVIS imaging system (40). The positive signal from background subtracted images was segmented and analyzed in Image Tool 3.0 (The University of Texas Health Science Center in San Antonio, TX) for integrated density as described by Long et al (40).

Toxicity of the CRAd agents in human liver tissue. Excess human liver not needed for diagnostic purposes was obtained from hepatectomy specimens following liver transplantation. To generate liver tissue slices, tissue was cut in consecutive $0.5-\mathrm{mm}$ thick slices using the Krumdieck tissue slicer. Sequential slices were then cultured in 24-well plates in RPMI medium supplemented with $10 \%$ bovine fetal serum, $100 \mathrm{U} / \mathrm{ml}$ penicillin, $100 \mu \mathrm{g} / \mathrm{ml}$ streptomycin and $5 \mu \mathrm{g} / \mathrm{ml}$ insulin. The tissue slices were infected with $500 \mathrm{vp} /$ cell of Adwt.F5/3, Ad-424.F5/3, CRAd-M.F5/3, CRAd-C.F5/3, CRAd-S.F5/3 or the non-replication control, Ad-CMV.Luc. $\mathrm{F} 5 / 3$. After 24 or $72 \mathrm{~h}$ of incubation, at $37^{\circ} \mathrm{C}$, in a humidified atmosphere of $95 \%$ air and $5 \% \mathrm{CO}_{2}$, DNA was extracted from liver tissue via the DNeasy Tissue Kit (Qiagen, Valencia, CA). DNA samples will be treated with DNase free RNase to remove possible RNA contamination. Ad E4 gene copy number was quantified using real-time PCR using human
B-actin as the control to normalize the E4 copy number as described elsewhere (41). The viral copy number was reported as E4 copies/ng DNA in this study.

Statistic analysis. The Student's t-test was employed for statistical analysis where $\mathrm{P}<0.05$ was considered to be statistically significant.

\section{Results}

Evaluation of capsid modifications in vitro. To screen Ad fiber modifications for viral infectivity enhancement, four Ad vectors with capsid modifications, RGD (24), F5/3 $(26,27)$, pk7 (28) and pk7RGD (28) were generated to target ovarian cancer cell lines via an alternative CAR-independent pathway as described previously. Four ovarian cancer cell lines, UCI101, SKOV3.ip1, HEY and OV4, were used in this study and maintained under standard conditions. All the Ad vectors had an identical Ad5 backbone containing a luciferase reporter gene driven by the CMV promoter, the exclusive difference being the incorporation of the alternative modifications in the Ad fiber region. The luciferase levels of the modified Ad vectors in the ovarian tumor cells were normalized to that of the Ad5-CMV.Luc (Ad5Luc) vector which had both the same backbone and the native Ad5 fiber. The data are shown in Fig. 1. The Ad vector with the F5/3 modification, Ad5-CMV.Luc.F5/3, exhibited the highest reporter activity among the four ovarian cancer cell lines tested. They were 260, 157, 153 and 2,645\% $($ mean $=183 \%)$ in UCI101, SKOV3.ip1, HEY and OV4 cell lines, respectively, when compared to that of the Ad5-CMV.Luc. The Ad vector carrying the RGD, pk7 and pk7RGD fiber modifications also exhibited high reporter activity in the OV4 cell lines, but reporter activity was similar to levels of Ad5-CMV.Luc in the other three cell lines. These data strongly argue that the F5/3 fiber modification should be an excellent candidate for viral infectivity enhancement in ovarian cancer cell directed CRAd agents.

F5/3 modified CRAds induce cytotoxicity in ovarian cancer cell lines. To evaluate the cytotoxicity of CRAd agents in ovarian cancer cell lines, we used the fiber F5/3 modified CRAd agents; Ad- $\Delta 24 . F 5 / 3$, CRAd-C.F/3, CRAd-M.F5/3 and CRAd-S.F5/3, based on the data from the Fig. 1. All these agents, including both 'positive' and 'negative' controls, had both the identical Ad5 backbone and the fiber modification, F5/3. The Ad- $\Delta 24$.F5/3 agent contained a partial deletion in the CR2 domain of the pRb-binding protein E1A (d1919-943) that is complemented in pRb-deficient tumor cells. Sole difference among these F5/3 modified CRAds was the promoter driving E1 gene expression. Among these were the promoters CXCR4, mesotheline and survivin. As an oncolytic anti-tumor agent, they were evaluated for their cell-killing effect in a variety of ovarian cancer cell lines. Cytotoxicity was evaluated after 10-day incubation via crystal violet staining (Fig. 2). While the replicationincompetent Ad5-Luc.F5/3 vector had no cytotoxic effect even at $100 \mathrm{vp} / \mathrm{cell}$, the F5/3 modified CRAds induced strong cytotoxicity in the all ovarian cell lines tested. Nearly $100 \%$ of cells were killed even at the dose, $10 \mathrm{vp} / \mathrm{cell}$, in 


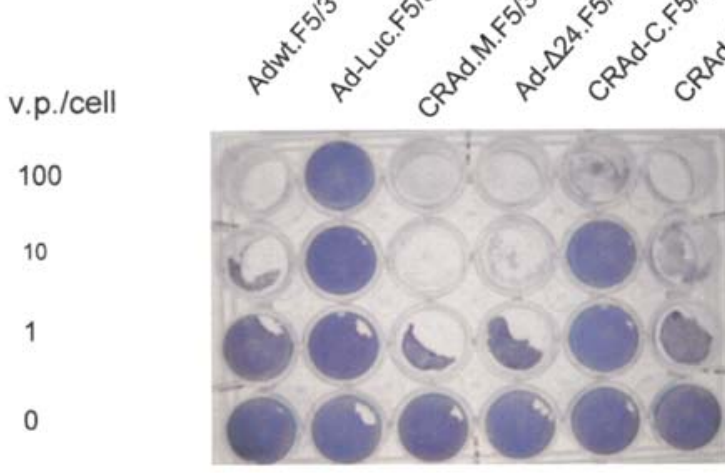

HEY

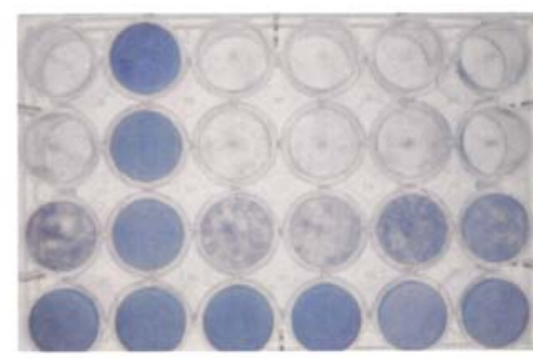

UCl-101
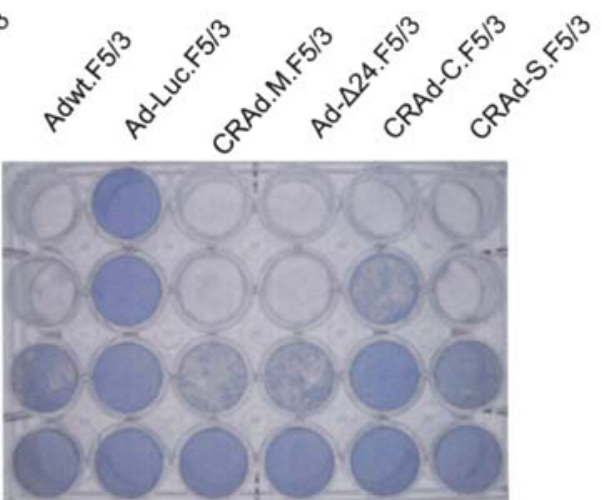

SKOV3ip1

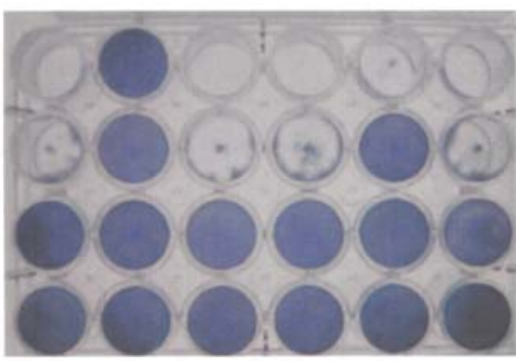

OV4

Figure 2. Oncolytic effect of CRAds in ovarian and HMEC cells. Ovarian cells and HMEC cells $\left(5 \times 10^{4}\right)$ were plated on 24-well plates, and infected with Ad vector (Ad5- L24.F5/3, CRAd-C.F5/3, CRAd-M.F5/3, or CRAd-S.F5/3 and a positive control, Adwt.F5/3; a non-replicative negative control, Ad-Luc.F5/3) at the indicated MOIs (100, 10,1 vp/cell) or mock-infected. After a 10-day incubation, cells were stained with crystal violet as described in Materials and methods.
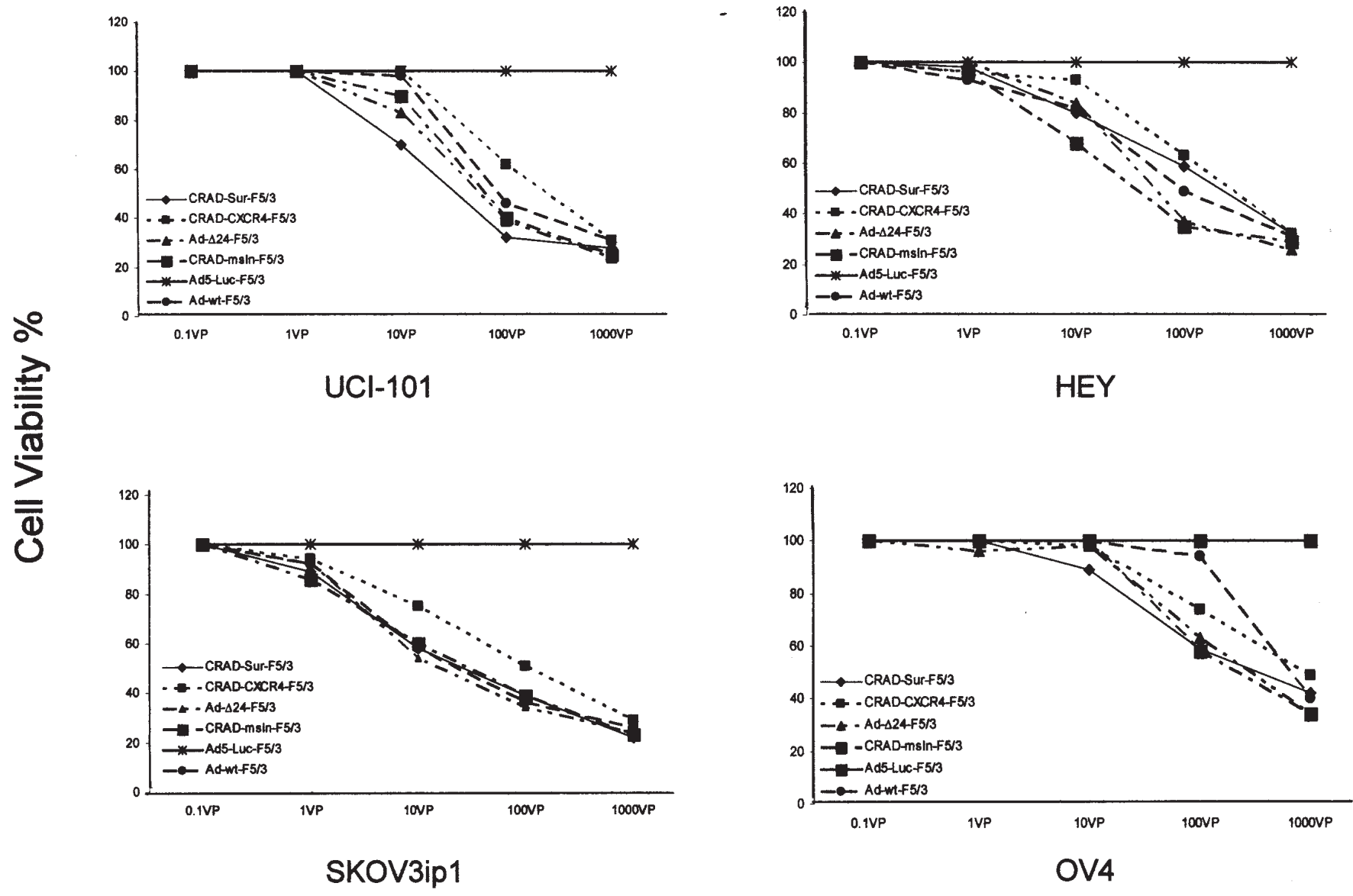

Figure 3. MTS cell viability assays. Ovarian cells $\left(5 \times 10^{3}\right)$ and HMEC cells were plated onto 24-well plates. Viability of ovarian carcinoma cells at day 5 after infection (MOI 1000, 100, 10,1,0) with Ad5- $224 . F 5 / 3$, CRAd-C.F5/3, CRAd-M.F5/3, or CRAd-S.F5/3 and both a positive control, Adwt.F5/3 and a non-replicative negative control, Ad-Luc.F5/3. Two sided error bars indicate \pm 1 standard deviation. 
A
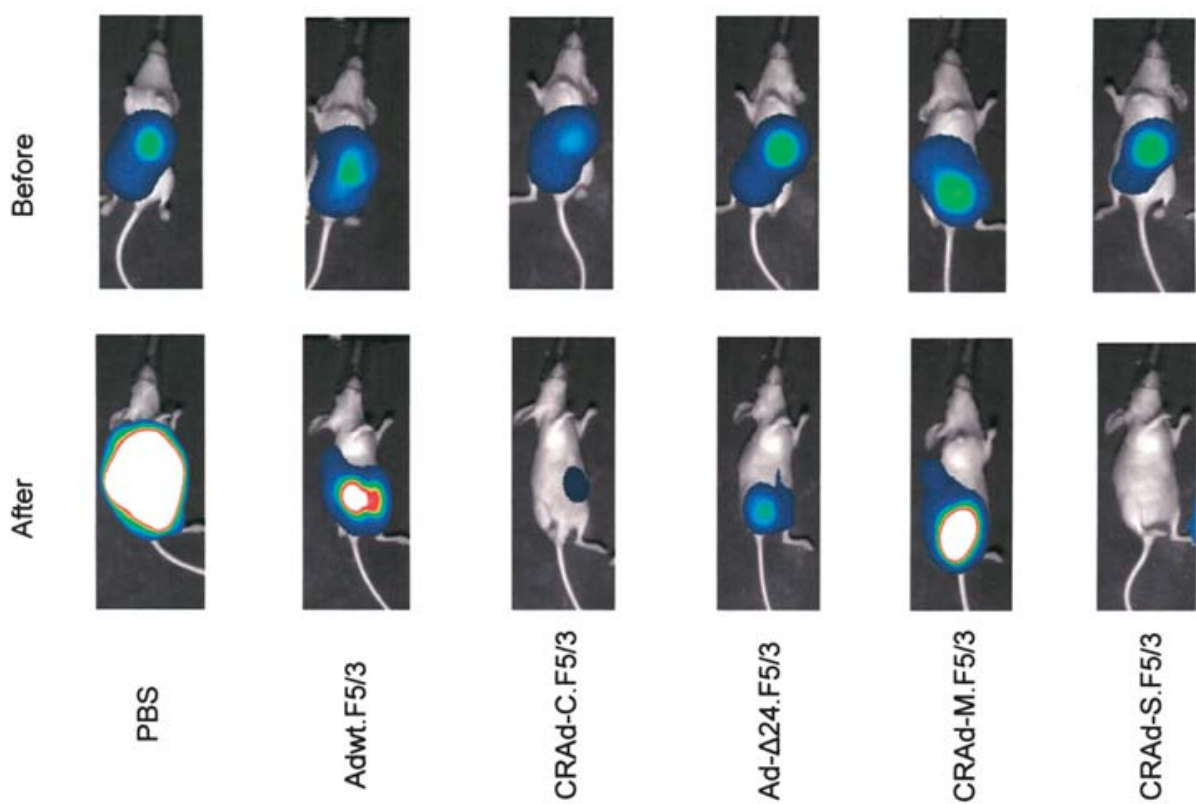

产

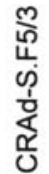

B

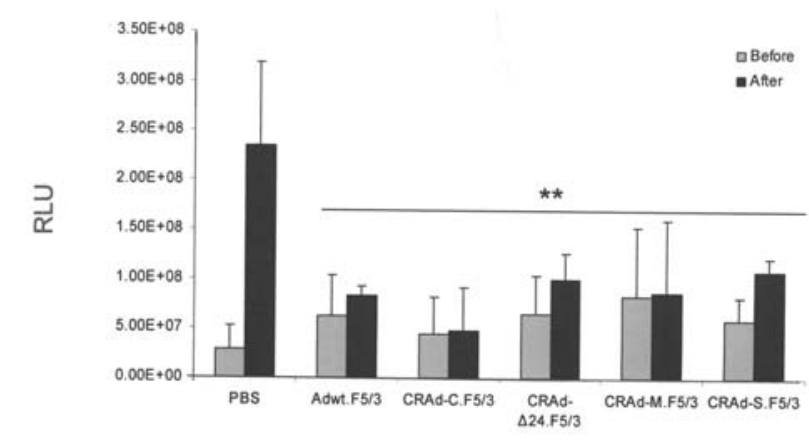

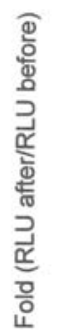

$\mathrm{C}$
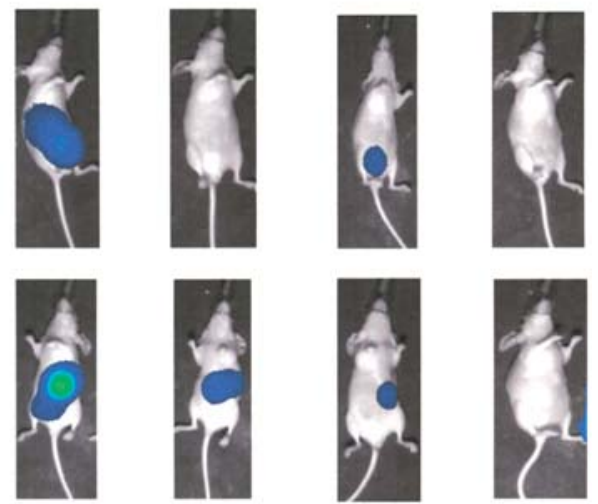

Treatment

Times
1

Figure 4. Tumor growth inhibition. SKOV3Luc cells $\left(1 \times 10^{7}\right)$ were inoculated i.p. and visible tumors were evident 5 days post injection. Ad vector and control ( $\left.1 \times 10^{9} \mathrm{vp}\right)$, including Adwt.F5/3, CRAd-C.F5/3, CRAd- $424 . F 5 / 3$, CRAd-M.F5/3, CRAd-S.F5/3 and PBS, in 200 $\mu 1$ volume was injected i.p. The injection continued once a week, 3 times. After inoculation and before treatment, the bioluminescent imaging was determined as described in Materials and methods. (A) Comparison of the bioluminescence imaging signals before and after treatment by group; (B) Anti-tumor effect of CRAd agents; and (C) The bioluminescence was seen to be absent in two mice following therapy.

the three cell lines HEY, SKOV3ip1 and ICI101 for CRAdM.F5/3, Ad- $\Delta 24 . F 5 / 3$ and CRAd-S.F5/3, with no significant difference. Only CRAd-C.F5/3 demonstrated a weak tumor killing effect, about one log dose higher than that of the other agents. Compared to the positive control, Adwt.F5/3, thus, three replication-competent Ad agents (Ad- $\Delta 24 . F 5 / 3$, CRAd-M.F5/3 and CRAd-S.F5/3) had higher activity in all four ovarian cancer cell lines tested.

Determination of cell viability with F5/3 modified replicationcompetent Ad agents. All cell lines and Ad agents were the same as in Fig. 2. A range of doses of Ad agents, i.e. 0, 1, 10,
100 and $1,000 \mathrm{vp} / \mathrm{cell}$, were used to infect the cells tested in 96-well plates. After a 5-day incubation, the cytotoxicity of the replication-competent Ad agents was evaluated using an MTS assay as described in Materials and methods. Although the cell viabilities were not significantly different among the four replication-competent adenovirus agents tested, at the dose of 1,000 vp/cell, slight differences were shown at a dose of $100 \mathrm{vp} /$ cell. A lower cell viability or stronger cell killing was seen with the CRA-S.F5/3 construct in the UCI101 cell line. CRAd-M.F5/3 was seen to be best in the HEY and Ad- $\Delta 24 . F 5 / 3$ in SKOV3.ip1 cells while CRAdM.F5/3 was superior in the OV4 tumor cell line. However, 
no one vector specifically targeted the majority of the ovarian cell lines (Fig. 3).

Comparison of anti-tumor effects of the replication-competent agents in vivo. The cell line, SKOV3Luc, with imaging capacity, was used in this experiment. A luciferase reporter gene was incorporated into the genome of the SKOV3 ovarian tumor cell line, leading it to permanently express the luciferase protein. We use this to monitor the anti-tumor effect of the replication-competent agents in this study. All mice were inoculated with $1 \times 10^{7}$ SKOV3Luc cells i.p., which had a viability rate over $98 \%$ as determined by using Trypan blue exclusion. Five days post inoculation, i.p. injection of $10^{9} \mathrm{vp}$ of Adwt.F5/3, Ad- $224 . F 5 / 3$, CRAd-C.F5/3, CRAd-M.F5/3, or CRAd-S.F5/3 plus a mimic PBS control were given once a week for 3 weeks. In PBS group all tested mice were sacrificed after second treatment; all mice in the other 4 groups were alive after the third treatment. We also used a genetic adenovirus labeling system to dynamically monitor the anti-tumor effect by fluorescent signal detection using a CCD camera. It was observed (Fig. 4A) that most mice in the treatment group demonstrated bioluminescence signals that were weaker after three treatments than that at zero time, before treatment. Further, the luminescent signals in the treatment groups were much weaker than that of the control, PBS group. In Fig. 4C, dynamic luminescence data were shown in two mice during treatment. One mouse received CRAd-C.F5/3 and the other CRAd-S.F5.3, the luminescent intensity, however, was seen to decrease during the three treatments. No signal could be detected after the third treatment in either mouse. Of note, we did not detect a signal after the first and third treatment in the CRAd-C.F5/3 group, but we detected it only after the second treatment. The mean luminescent signals for the five animals from each group are plotted in Fig. 4B. Comparing the data before and after treatment, the signal increased in all six groups. However, the signal intensity increased much more in the control group than in CRAd treatment groups. It was $>8$ fold increased in the control PBS group, however, $<2$-fold increased in replication virus groups with significant difference. Thus, it is demonstrated that the ovarian tumor (SKOV3Luc) growth in mouse peritoneal was inhibited by injected CRAd agent i.p.

The replication rates of CRAd vectors in human liver slices. To demonstrate replication specificity of the CRAd agents, we examined all CRAd agents, including Ad- $\Delta 24 . F 5 / 3$, CRAd-M.F5/3, CRAd-C.F5/3, CRAd-S.F5/3 and both the positive (Adwt.F5/3) and negative (Ad-Luc.F5/3) controls, in human liver slices. The CRAd agents replicated in human liver and amplified the viral particles in this tissue. We quantified the viral particle load using real-time PCR (a sensitive technique) and compared the viral particles at day 3 with that at day 1 . The replication rates of the CRAd agents were presented as the increased 'fold' of the viral particles at day 3 divided by that of day 1 . A higher viral replication rate of the agent is reflected by a higher 'fold' and thus risk of a higher toxicity in liver tissue. From Fig. 5, we can see the increased 'folds' were $8.7,4.1,20.7,7.2,9.5$ and 4.9 for Adwt.F5/3, Ad.Luc.F5/3, CRAdM.F5/3 Ad- $424 . F 5 / 3$,

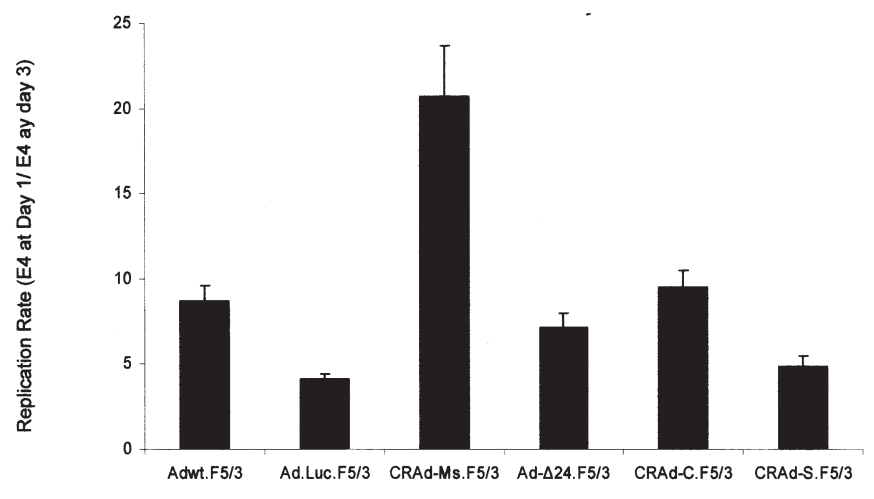

Figure 5. Viral replication of CRAd agents in human liver slices. Human liver slices were infected with $500 \mathrm{vp} /$ cell of Ad.Luc.F5/3, Adwt.F5/3, CRAd-C.F5/3, CRAd- $\Delta 24 . F 5 / 3$, CRAd-M.F5/3 or CRAd-S.F5/3. One and three days following infection, DNA was extracted from cells. Adenovirus E4 copy number and the $\beta$-actin gene were detected by using real-time PCR as described. B-actin was used as a housekeeping gene to normalize E4 copy number. The replication rate of CRAd agents was presented as 'fold' change in which the $\mathrm{E} 4$ copies at day $3 /$ the $\mathrm{E} 4$ copies at day $1 .{ }^{* *} \mathrm{P}<0.01$.

CRAd-C.F5/3 and CRAd-S.F5/3, respectively. CRAd-S.F5/3 showed the lowest viral replication rate (4.9-fold), similar to the level in the non-replication Ad.Luc.F5/3 control virus (4.1-fold). The CRAd-M.F5/3 had the highest viral replication rate (20.7-fold) which was four times higher than that of the CRAd-S.F5/3. The positive control Adwt.F5/3 had an intermediate level of viral replication. Thus, we find two CRAd agents, Ad- $124 . F 5 / 3$ and CRAd-S.F5/3, have low viral replication rate in human liver tissue that relates to low viral toxicity.

\section{Discussion}

In this study, we evaluated efficacies of multiple CRAd agents in ovarian cancer both in vitro and in vivo. Our data show: i) the best Ad fiber modification (F5/3) to target ovarian cancer in vitro at transductional level via a CARindependent pathway; ii) the CRAd agents with higher tumor killing effect and tumor growth inhibition for ovarian cancer both in vitro and in vivo at transcriptional level; and iii) the CRAd agents with the lowest viral replication rate in human liver tissue, related to low toxicity of the CRAd agent. We used these criteria to evaluate multiple CRAd agents. Both CRAd- $\Delta 24$.F5/3 and CRAd-S.F5/3 were shown to be excellent candidates for targeting ovarian cancer.

Many CRAd agents with various promoters have previously been screened in ovarian cancer (38). Five oncolytic adenovirus candidates, included RGDCRAdcox2R, Ad5VEGF1, Ad5/3VEGF1, Ad5- $224 \mathrm{RGD}$, Ad5/3- 124 , were analyzed for ovarian cancer therapy. The study included primary tumor tissue, spheroids, and an orthotopic model of human ovarian cancer to evaluate these CRAds that show promise for treat-ment of peritoneal matastatic overian cancer. Because of the different fiber modification in these CRAd agents, thus, it is difficult to evaluate the viral replication and tumor cell killing at the transductional or transcriptional level. Also, the toxicity analysis of the CRAd 
agents has not been fully examined and reported. In this study, we compared four fiber modifications, specifically RGD, F5/3, pk7 and RGDpk7, in ovarian cancer cell lines. Fig. 1 clearly shows that the F5/3 chimera is the best fiber modification in all four tested ovarian cancer cell lines. Compared to the reporter gene expression level of Ad5CMV.Luc (a positive control with a native Ad5 knob without modification) there were $260,157,153$ and 2,645\% levels, respectively, in UCI101, SKOV3ip1, HEY and OV4 cell lines. These, in turn, were higher than that of the other three fiber modifications. As the F5/3 modification was seen to be the best to enhance the viral infectivity in ovarian cancer cell lines, we used it as the fiber modification in all tested CRAd agents in order to maintain an identical transductional level among all tested CRAd agents.

Conditionally replicative adenovirus vectors can be divided into 2 generations, arbitrarily designated type I and type II. The type I CRAd agents target biological factors known to be modified in cancer cells. Examples of type I CRAd agents are d11520 (Onyx-015) (7) and Ad5- $\Delta 24$, which is used in this study (19). Since this gene product binds the cellular retinoblastoma $(\mathrm{Rb})$ protein to induce S-phase entry, these CRAd agents have limited ability to overcome the G1-S checkpoint in normal cells. In contrast, these viruses replicate efficiently in cells with mutant $\mathrm{Rb}$, such as tumor cells, where this interaction is unnecessary. The Rb-p16 pathway has been reported to be defective in many human tumors, these CRAd agents thus replicate with high specificity in maglignant cells, including those of ovarian origin. The CRAd agent, Ad- $224 . R G D$, is currently being evaluated in clinical trials of ovarian cancer at UAB. The other three CRAd agents used in this study all belong to the type II CRAd agent in which the E1A gene is under the control of the TSP, the CXCR4, mesothelin or survivin promoter, respectively. All these CRAd agents incorporated a fiber modification, F5/3, to enhance viral activity. In addition, the F5/3 modification assures identical transductional level in all CRAd agents, thus, it maximizes the ability to specifically evaluate viral replication of these agents in ovarian cancer cell lines in vitro and in vivo.

We compared in head-to-head manner four CRAd agents. In cytotoxicity assay, the three replication-competent $\mathrm{Ad}$ agents (CRAd-M.F5/3, Ad- $224 . F 5 / 3$ and CRAd-S.F5/3) had a higher (one $\log$ ) activity than that of the positive Adwt.F5/3 control in all four ovarian cancer cell lines tested. However, no significant difference from each other was noted (Fig. 2). In addition, although cell viabilities were not significantly different among the four CRAd agents tested at the dose of $1,000 \mathrm{vp} / \mathrm{cell}$, slight differences were evident at the dose of $100 \mathrm{vp} / \mathrm{cell}$. Lower cell viability or stronger cell killing was seen with CRA-S.F5/3 in the UCI101 cell line, CRAdM.F5/3 in HEY, Ad- $\Delta 24 . F 5 / 3$ in SKOV3ip 1 and CRAdM.F5/3 in the OV4 cell line (Fig. 3). Both in vitro experiments (Figs. 2 and 3) provide no evidence as to which CRAd agent more specifically targets ovarian cancer cell lines.

To further screen the four CRAd agents in ovarian cancer cells in vivo, an imaging cell line, SKOV3Luc, was used in a later experiment. A luciferase reporter gene was incorporated into the genome of SKOV3 cells allowing it to express the reporter gene products. We thus used this imaging modality to monitor the anti-tumor effect of the replication-competent agents in the study by bioluminescent imaging. This imaging relied upon a luciferase (Luc) reporter gene from the North American firefly Photinus pyralis. This reporter has several advantages compared to other imaging systems: i) the technique is sensitive $\left(10^{-15}-10^{-17}\right.$ mole of luciferase/l are detectable in vivo (42); ii) bioluminescent imaging using a cooled CCD camera does not require great technical expertise; iii) it is faster and less expensive than many other imaging techniques; iv) there is low background; and v) it allows a non-invasive analysis for evaluating anti-tumor effects of the best CRAd agent for preclinical evaluation.

A representative example from each group was selected for similar luminescent signal intensity before treatment began as shown in Fig. 4A. All mice were alive after the third treatment, but within the PBS group all 5 mice died after the second treatment. Comparing the bioluminescent imaging signal of the treatment group to that of the control group, PBS, the signal intensity is much weaker in the treatment groups. It was $>8$-fold increased in the control PBS group, however, $<2$-fold increased in replication virus groups $(\mathrm{P}<0.01)$. Although significant difference was not found between the CRAd agent groups and positive control (Adwt.F5/3) (Fig. 4B), it was clear that CRAd agents inhibited tumor growth, but we still could not verify which was the best one. Bioluminescent imaging could not be detected after the third treatment in two mice, one receiving the CRAd-C.F5/3 construct and the other from the CRAS.F5/3 group (Fig. 4C). The bioluminescent imaging signal disappearance, however, should not be taken as evidence that the tumor was eliminated, because the signal was not detected in the CRAd-C.F5/3 group either after the first treatment.

Infection with adenovirus causes profound changes in host-cell macromolecular synthesis that ultimately leads to cell death. Virion fiber protein inhibits macromolecular synthesis when applied directly to cells bearing the adenovirus receptor (43). Cell-specific DNA synthesis exports cellular mRNAs from the nucleus to the cytoplasm, and cell-specific translation is inhibited after infection. The precise mechanisms are still not completely understood. The main damage to the host, however, is that the majority $(>95 \%)$ of viruses released into the blood stream and localized to the liver $(24,44)$ lead to host toxicity. To mimic the in vivo condition of the human host, we used human liver slices instead of murine liver, as we had in previous studies to rigorously evaluate the activity of the promoters which control viral replication in CRAd agents. The replication ratios of Ad vectors in human liver slices are shown in Fig. 5. The highest is CRAd-M.F5/3 (20.7-fold) and the lowest is CRAd-S.F5/3 (4.9-fold) when comparing the Ad particles 3 days post infection to that of 1 day post infection. The replication rate of CRAd-M.F5/3 is extremely high in human liver, thus, higher toxicity to human liver is anticipated. Although, CRAd-M.F5/3 had a similar tumor cell killing effect to that of CRAd- $\Delta 24 . F 5 / 3$ and CRAd-S.F5/3, the CRAd-M.F5/3 is not a good candidate for treatment of ovarian cancer due to higher replication rate in human liver and thus potential higher toxicity. The CRAd-S.F5/3 agent had presumed lower toxicity within the human liver due to a 
lower viral replication rate (4.9-fold) which was similar to that of the non-replication control, Ad-Luc.F5/3 (4.1-fold). Another CRAd agent, CRAd $\Delta 24 . F 5 / 3$, had a 7.2-fold replication rate which was lower than that of the positive control, Adwt. F5/3 (8.7-fold) and higher than that seen with the non-replicative viral control Ad.Luc.F5/3 (4.1-fold).

In summary, this study sought to analyze Ad vector transductional activity, transcriptional activity and toxicity in vitro and in vivo. Both the CRAd- $\Delta 24 . F 5 / 3$ and CRAdS.F5/3 appear to be good CRAd agent candidates for the treatment of ovarian cancer because they have high transductional, transcriptional and tumor cell killing activities in human ovarian cancer cell lines with lower toxicity in human liver tissue. Clinical trials will ultimately determine their safety and efficacy.

\section{Acknowledgments}

We thank Drs F. Li, NL Michael and V. Kranykh for providing vectors for this study. The study was supported by the National Institute of Health grants: 5RO1 CA083821, 2R56 CA094084, and Korea Research Fundation Grant funded by the Korean Government (MOEHRD) (KRF-2006214-E00017).

\section{References}

1. Jemal A, Siegel R, Ward E, et al: Cancer Statistics. CA Cancer J Clin 56: 106-130, 2006.

2. Spriggs D: Optimal sequencing in the treatment of recurrent ovarian cancer. Gynecol Oncol 90: S39-S44, 2003.

3. Armstrong DK: Relapsed ovarian cancer: challenges and management strategies for a chronic disease. Oncologist 7 (Suppl 5): 20-28, 2002.

4. Herzog TJ: Recurrent ovarian cancer: how important is it to treat to disease progression? Clin Cancer Res 10: 7439-7449, 2004.

5. Webb HE and Smith CE: Viruses in the treatment of cancer. Lancet 1: 1206-1208, 1970.

6. Barker DD and Berk AJ: Adenovirus proteins from both E1B reading frames are required for transformation of rodent cells by viral infection and DNA transfection. Virology 156: 107-121, 1987.

7. Bischoff JR, Kirn DH, Williams A, et al: An adenovirus mutant that replicates selectively in p53-deficient human tumor cells. Science 274: 373-376, 1996.

8. Harada JN and Berk AJ: p53-Independent and -dependent requirements for E1B-55K in adenovirus type 5 replication. $\mathrm{J}$ Virol 73: 5333-5344, 1999.

9. Ganly I, Kirn D, Eckhardt S, et al: A phase I study of Onyx015, an E1B attenuated adenovirus, administered intratumorally to patients with recurrent head and neck cancer. Clin Cancer Res 6: 798-806, 2000.

10. Mulvihill S, Warren R, Venook A, et al: Safety and feasibility of injection with an E1B-55 kDa gene-deleted, replicationselective adenovirus (ONYX-015) into primary carcinomas of the pancreas: a phase I trial. Gene Ther 8: 308-315, 2001.

11. Rein DT, Breidenbach M, Kirby TO, et al: A fiber-modified, secretory leukoprotease inhibitor promoter-based conditionally replicating adenovirus for treatment of ovarian cancer. Clin Cancer Res 11: 1327-1335, 2005.

12. Vasey PA, Shulman LN, Campos S, et al: Phase I trial of intraperitoneal injection of the E1B-55-kd-gene-deleted adenovirus ONYX-015 (dl1520) given on days 1 through 5 every 3 weeks in patients with recurrent/refractory epithelial ovarian cancer. J Clin Oncol 20: 1562-1569, 2002.

13. Makower D, Rozenblit A, Kaufman H, et al: Phase II clinical trial of intralesional administration of the oncolytic adenovirus ONYX-015 in patients with hepatobiliary tumors with correlative p53 studies. Clin Cancer Res 9: 693-702, 2003.

14. Fueyo J, Gomez-Manzano C, et al: A mutant oncolytic adenovirus targeting the $\mathrm{Rb}$ pathway produces anti-glioma effect in vivo. Oncogene 19: 2-12, 2000.
15. Page JG and Tian B: Identifying the safety profile of a novel infectivity-enhanced conditionally replicative adenovirus, Ad5delta24-RGD, in anticipation of a phase I trial for recurrent ovarian cancer. Am J Obstet Gynecol 196: 389 e1-10, 2007.

16. Bauerschmitz GJ, Lam JT, Kanerva A, et al: Treatment of ovarian cancer with a tropism modified oncolytic adenovirus. Cancer Res 62: 1266-1270, 2002.

17. Lamfers ML, Grill J, Dirven CM, et al: Potential of the conditionally replicative adenovirus Ad5-Delta24RGD in the treatment of malignant gliomas and its enhanced effect with radiotherapy. Cancer Res 62: 5736-5742, 2002.

18. Suzuki K, Alemany R, Yamamoto M, et al: The presence of the adenovirus E3 region improves the oncolytic potency of conditionally replicative adenoviruses. Clin Cancer Res 8: 3348-3359, 2002.

19. Suzuki K, Fueyo J, Krasnykh V, et al: A conditionally replicative adenovirus with enhanced infectivity shows improved oncolytic potency. Clin Cancer Res 7: 120-126, 2001.

20. Hemmi S, Geertsen R, Mezzacasa A, et al: The presence of human coxsackievirus and adenovirus receptor is associated with efficient adenovirus-mediated transgene expression in human melanoma cell cultures. Hum Gene Ther 9: 2363-2373, 1998.

21. Miller CR, Buchsbaum DJ, Reynolds PN, et al: Differential susceptibility of primary and established human glioma cells to adenovirus infection: targeting via the epidermal growth factor receptor achieves fiber receptor-independent gene transfer. Cancer Res 58: 5738-5748, 1998.

22. Reynolds PN: Targeting gene delivery for pulmonary disease. In: Gene Therapy for Diseases of the Lung. Vol 169. Albelda SM (ed), Marcel Dekker pp119-144, 2002.

23. Krasnykh V, Dmitriev I, Mikheeva G, et al: Characterization of an adenovirus vector containing a heterologous peptide epitope in the HI loop of the fiber knob. J Virol 72: 1844-1852, 1998.

24. Dmitriev I, Krasnykh V, Miller CR, et al: An adenovirus vector with genetically modified fibers demonstrates expanded tropism via utilization of a coxsackievirus and adenovirus receptorindependent cell entry mechanism. J Virol 72: 9706-9713, 1998.

25. Wickham TJ, Mathias P, Cheresh DA, et al: Integrins alpha v beta 3 and alpha $\mathrm{v}$ beta 5 promote adenovirus internalization but not virus attachment. Cell 73: 309-319, 1993.

26. Stevenso SC, Rollence M, Marshall-Neff J, et al: Selective targeting of human cells by a chimeric adenovirus vector containing a modified fiber protein. J Virol 71: 4782-4790, 1997.

27. Von Seggern DJ, Huang S, Fleck SK, et al: Adenovirus vector pseudotyping in fiber-expressing cell lines: improved transduction of Epstein-Barr virus-transformed B cells. J Virol 74: 354-362, 2000.

28. Wu H, Seki T, Dmitriev I, et al: Double modification of adenovirus fiber with RGD and polylysine motifs improves coxsackievirus-adenovirus receptor-independent gene transfer efficiency. Hum Gene Ther 13: 1647-1653, 2002.

29. Lee SE, Jin RJ, Lee SG, et al: Development of a new plasmid vector with PSA-promoter and enhancer expressing tissuespecificity in prostate carcinoma cell lines. Anticancer Res 20: 417-422, 2000.

30. Huber BE, Richards CA and Krenitsky TA: Retroviral-mediated gene therapy for the treatment of hepatocellular carcinoma: an innovative approach for cancer therapy. Proc Natl Acad Sci USA 88: 8039-8043, 1991.

31. Yamamoto M, Davydova J, Wang M, et al: Infectivity enhanced, cyclooxygenase-2 promoter-based conditionally replicative adenovirus for pancreatic cancer. Gastroenterology 125: 1203-1218, 2003.

32. Adachi Y, Reynolds PN, Yamamoto M, et al: Midkine promoterbased adenoviral vector gene delivery for pediatric solid tumors. Cancer Res 60: 4305-4310, 2000.

33. Goldman CK, Kendall RL, Cabrera R, et al: Paracrine expression of a native soluble vascular endothelial growth factor receptor inhibits tumor growth, metastasis and mortality rate. Proc Natl Acad Sci USA 95: 8795-8800, 1998.

34. Barker SD, Coolidge CJ, Kanerva A, et al: The secretory leukoprotease inhibitor (SLPI) promoter for ovarian cancer gene therapy. J Gene Med 5: 300-310, 2003.

35. Uchino J, Takayama K, Harada A, et al: Infectivity enhanced, hTERT promoter-based conditionally replicative adenoviruses are useful for SCLC treatment. Cancer Gene Ther 12: 737-748, 2005 . 
36. Zhu ZB, Makhija SK, Lu B, et al: Transcriptional targeting of adenoviral vector through the CXCR4 tumor-specific promoter. Gene Ther 11: 645-648, 2004

37. Zhu ZB, Makhija SK, Lu B, et al: Transcriptional targeting of tumors with a novel tumor-specific survivin promoter. Cancer Gene Ther 11: 256-262, 2004.

38. Lam JT, Kanerva A, Bauerschmitz GJ, et al: Inter-patient variation in efficacy of five oncolytic adenovirus candidates for ovarian cancer therapy. J Gene Med 6: 1333-1342, 2004.

39. Fueyo J, Gomez-Manzano C, Yung WK, et al: Overexpression of E2F-1 in glioma triggers apoptosis and suppresses tumor growth in vitro and in vivo. Nat Med 4: 685-690, 1998.

40. Long P, Le HN, Dmitriev IP, et al: Dynamic monitoring of oncolytic adenovirus in vivo by genetic capsid labeling. J Natl Cancer Inst 98: 203-214, 2006.
41. Zhu ZB, Makhija SK, Lu B, et al: Incorporating the survivin promoter in an infectivity enhanced CRAd-analysis of oncolysis and anti-tumor effects in vitro and in vivo. Int J Oncol 27 : 237-246, 2005.

42. Massoud TF and Gambhir SS: Molecular imaging in living subjects: seeing fundamental biological processes in a new light. Genes Dev 17: 545-580, 2003.

43. Levine AJ and Ginsberg HS: Mechanism by which fiber antigen inhibits multiplication of type 5 adenovirus. J Virol 1: 747-757, 1967.

44. Reynolds P, Dmitriev I and Curiel DT: Insertion of an RGD motif into the HI loop of adenovirus fiber protein alters the distribution of transgene expression of the systemically administered vector. Gene Ther 6: 1336-1339, 1999. 